\section{Correlation between Kidney Function and Sonographic Texture Features after Allograft Transplantation with Corresponding to Serum Creatinine: A Long Term Follow-Up Study}

\author{
Abbasian Ardakani A. ${ }^{1 \oplus}$, Sattar A. R. ${ }^{2}$, Abolghasemi J. ${ }^{3}$, \\ Mohammadi A.2*(อ)
}

\begin{abstract}
Background: The ability to monitor kidney function after transplantation is one of the major factors to improve care of patients.
\end{abstract}

Objective: Authors recommend a computerized texture analysis using run-length matrix features for detection of changes in kidney tissue after allograft in ultrasound imaging.

Material and Methods: A total of 40 kidney allograft recipients (28 male, 12 female) were used in this longitudinal study. Of the 40 patients, 23 and 17 patients showed increased serum creatinine ( $\mathrm{sCr}$ ) (increased group) and decreased $\mathrm{sCr}$ (decreased group), respectively. Twenty run-length matrix features were used for texture analysis in three normalizations. Correlations of texture features with serum creatinine $(\mathrm{sCr})$ level and differences between before and after follow-up for each group were analyzed. An area under the receiver operating characteristic curve $\left(A_{z}\right)$ was measured to evaluate potential of proposed method.

Results: The features under default and 3sigma normalization schemes via linear discriminant analysis (LDA) showed high performance in classifying decreased group with an $\mathrm{A}_{\mathrm{z}}$ of 1 . In classification of the increased group, the best performance gains were determined in the 3 sigma normalization schemes via LDA with an $\mathrm{A}_{\mathrm{z}}$ of 0.974 corresponding to $95.65 \%$ sensitivity, $91.30 \%$ specificity, $93.47 \%$ accuracy, 91.67\% PPV, and $95.45 \%$ NPV.

Conclusion: Run-length matrix features not only have high potential for characterization but also can help physicians to diagnose kidney failure after transplantation.

Citation: Abbasian Ardakani A, Sattar AR, Abolghasemi J, Mohammadi A. Correlation between Kidney Function and Sonographic Texture Features after Allograft Transplantation with Corresponding to Serum Creatinine: A Long Term Follow-Up Study. J Biomed Phys Eng. 2020;10(6):713-726. doi: 10.31661/jbpe.v0i0.928.

\section{Keywords}

Decision Making; Computer-Assisted; Kidney Transplantation; Pattern Recognition System; Ultrasonography

\section{Introduction}

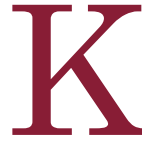
idney transplantation is an elective treatment for patients with chronic kidney disease (CKD) and its progression, end-stage renal disease (ESRD) [1]. Despite recent improvements in treatment, acute rejection leads to graft failure in $12 \%$ of cases [2]. The serum
${ }^{1} \mathrm{PhD}$, Department of Medical Physics, School of Medicine, Iran University of Medical Sciences,

Tehran, Iran

${ }^{2} \mathrm{MD}$, Department of

Vascular and Interven-

tional Radiology, School

of Medicine, Namazi

Hospital, Shiraz Univer-

sity of Medical Sciences,

Shiraz, Iran

${ }^{3} \mathrm{PhD}$, Department of

Biostatistics, School

of Public Health, Iran

University of Medical Sci-

ences, Tehran, Iran

*Corresponding author: A. Mohammadi

Department of Vascu-

lar and Interventional

Radiology, School of

Medicine, Namazi Hos-

pital, Shiraz University

of Medical Sciences,

Shiraz, Iran

E-mail: Afshin.mohdi@

Gmail.com

Received: 16 April 2018

Accepted: 17 June 2018 
creatinine $(\mathrm{sCr})$ concentration is commonly used as a marker for evaluating kidney graft functioning. Chronic allograft nephropathy appears with increasing interstitial fibrosis and tubular atrophy increasing creatinine [3-5]. It seems that interstitial fibrosis and tubular atrophy can change properties of medical images.

Ultrasonography is the first chosen technique to evaluate kidney function after allograft transplants owing to being in real time, non-invasive, radiation-free, widely available and convenient [6]. Different tissues have different textures in an ultrasound image, which is made of diverse gray-level intensities. Texture can be simply conceived by human eyes though there is no accurate mathematical definition for it. Various coarse, fine, and smooth patterns as well as spatial variations in the pixel intensities of objects describe an image texture. Although it is difficult to interpret complex patterns, visual inspection can provide a detection of structural abnormalities within an ultrasound image. Yet, a computerized texture analysis has been proposed to improve decision of physicians and reduce patient pain besides avoiding repeat biopsy and laboratory tests. In conventional ultrasound imaging, computerized texture analysis can mathematically detect pathological changes which are not perceivable by human eyes $[7,8]$.

Differentiation of kidney diseases [9-12] and monitoring the progression of CKD stages [13, 14] have been possible through texture analysis (TA) in recent studies. It is shown that TA of ultrasound image can differentiate normal cases from medical renal disease (MRD) and cortical cyst $[9,15,16]$ as well as to diagnose bacterial infections [10]. Additionally, more information on this method was provided in this research. As mentioned before, interstitial fibrosis and tubular atrophy are caused via allograft rejection. Thus, these changes can have an influence on kidney texture features.

This study aims to investigate performance of texture features to monitor kidney function after transplanting among recipients. This is the first study using texture analysis to evaluate kidney allograft recipients after long-term follow-up.

\section{Material and Methods}

Study Design and image acquisition Kidney allograft recipients with 3 years follow-up information were considered for enrollment in this longitudinal study. The inclusion criteria are as following: 1) each of the patients received an allograft from a living donor at the Namazi Hospital. 2) All patients were treated with similar immunosuppressive drugs based on steroids, mycophenolate, mofetil, and tacrolimus. 3) The $\mathrm{sCr}$ levels were measured by the Jaffe method. 4) $\mathrm{Pa}$ tients were biopsy proven chronic allograft nephropathy. Furthermore, the exclusion criteria are obtained to avoid potential confusion. The exclusion criteria are significant arrhythmia, large perinephric collections, HIV-positive, chronic inflammatory, diabetes, an inter current infection, re-transplant, hydronephrosis, all causes of increased $\mathrm{sCr}$ such as pre- or post-renal azotemia and individuals who were alcoholics and/or smokers.

In this study, longitudinal view of ultrasound images were acquired by Accuvix WS80 sonography device (Medison, Seoul, Korea) equipped with 7-11 MHz linear transducer. Ultrasound images were taken before and at follow-up for each recipient. $\mathrm{sCr}$ concentrations were determined a few moments before sonography examination. All ultrasound images were taken in same condition and the mid-pole of kidneys was selected for TA.

Body mass index (BMI) was calculated $(\mathrm{kg} /$ $\mathrm{m}^{2}$ ) during a physical examination. BMI was categorized as underweight, normal weight, overweight, obese class I, obese class II and extremely obese class III [17]. Ultrasound images, $\mathrm{BMI}$ and $\mathrm{sCr}$ were acquired prior to the allograft procedure and at 3 years follow-up of it. 
Kidney Function Assessment Using Texture Analysis

ROI Selection and Run-length $\mathrm{Ma}-$ trix Features Extraction

One 2-D region of interest (ROI) was selected for each image of the kidney allograft; hence 80 non-overlap ROIs (total before and at follow-up) were selected for TA. To prevent any bias in ROIs selection, they were defined on images by software and checked by a single experienced radiologist.

This study proposed TA using Run-length Matrix (RLM) to find differences of patients image which were divided into 2 groups: before and after 3 years follow-up. RLM evaluates the image in a specific direction to find pixels with the same intensity. Matrix element $(i, j)$ indicates continuous pixel $(j)$ in the kidney region with the same intensityi (i) in the specific direction. In this study, five RLM parameters were used to describe kidney finction: short-run emphasis (SRE), long-run emphasis (LRE), gray-level nonuniformity (GLNU), run-length nonuniformity (RLNU), and fraction of the image in runs (Fraction). They are defined as:

$$
\begin{aligned}
& \mathrm{SRE}=\sum_{i=1}^{N_{a}} \sum_{j=1}^{N_{r}} \frac{P(i . j)}{j^{2}} / \sum_{i=1}^{N_{a}} \sum_{j=1}^{N_{r}} P(i . j) \\
& \mathrm{LRE}=\sum_{i=1}^{N_{a}} \sum_{j=1}^{N_{r}} j^{2} P(i . j) / \sum_{i=1}^{N_{a}} \sum_{j=1}^{N_{r}} P(i . j) \\
& \mathrm{GLNU}=\sum_{j=1}^{N_{r}}\left(\sum_{i=1}^{N_{a}} P(i . j)\right)^{2} / \sum_{i=1}^{N_{a}} \sum_{j=1}^{N_{r}} P(i . j) \\
& \mathrm{RLNU}=\sum_{i=1}^{N_{a}}\left(\sum_{j=1}^{N_{r}} P(i . j)\right)^{2} / \sum_{i=1}^{N_{a}} \sum_{j=1}^{N_{r}} P(i . j) \\
& \text { Fraction }=\sum_{i=1}^{N_{a}} \sum_{j=1}^{N_{r}} P(i . j) / \sum_{i=1}^{N_{a}} \sum_{j=1}^{N_{r}} j P(i . j)
\end{aligned}
$$

Where $\mathrm{N}_{\mathrm{a}}$ and $\mathrm{N}_{\mathrm{r}}$ are the number of gray levels in the image and different run lengths occurring, respectively. These five parameters are calculated in four directions, including horizontal, vertical, 45-degree (45 Dgr), and 135-degree (135 Dgr).

Pixel Normalization and Standardization

The 20 RLM features were measured for each region in three normalizations, including default, 3-sigma and 1\%-99\% normalizations. In the default scheme, the intensity of pixels in the kidney region was not changed. However, in 3-sigma and 1\%-99\% normalization, the pixels intensity of kidney region were normalized in the range of $\mu \pm 3 \sigma$ and between $1 \%$ and $99 \%$ gray-level of total intensity. Where $\mu$ and $\sigma$ are mean and standard deviation of intensities within the kidney images, respectively.

Before analysis, the RLM features were standardized as follows:

$$
\mathrm{x}_{\mathrm{i}}^{\prime}=\frac{\mathrm{x}_{\mathrm{i}}-\mu_{\mathrm{i}}}{\mathrm{o}_{\mathrm{i}}}
$$

where $x_{i}$ and $x_{i}^{\prime}$ are RLM feature before and after standardization process, respectively, $\mu_{i}$ is mean and $\sigma_{i}$ is standard deviation of the $i$-th feature. The standard and nonstandard features were analyzed to find good outcome.

\section{Statistical Analysis and Classifi- cation}

Data were tested for normality by the Kolmogorov-Smirnov test. Paired samples t-test was obtained to find differences between before and after follow-up for each group. An area under the receiver operating characteristic $(\mathrm{ROC})$ curve $\left(\mathrm{A}_{\mathrm{z}}\right)$ was obtained to evaluate performance of classification for before and after follow-up for each group. The two-tailed Pearson correlation test was implemented to find correlation between $\mathrm{sCr}$ level and RLM parameters. A P-value of $<0.05$ was considered statistically significant.

Texture features that showed a significant difference were utilized for computerized multi parameter texture analysis (MPTA). In this regard, linear discriminant analysis (LDA) and principle component analysis (PCA) were 
used to analyze the data and map them in to a lower dimension. In the final step, the data were classified by $\mathrm{K}-\mathrm{NN}(\mathrm{K}=1)$ classifier. The ROC analysis was employed to compare the performances of classification in different data analysis strategies. Figure 1 shows the computer-aided diagnosis (CAD) processing steps.

SPSS version 19 was used for statistical analyses. 95\% confidence intervals for sensitivity, specificity, positive predictive value (PPV) and negative predictive value (NPV) were computed by R software, version 3.3.1 (R Project for Statistical Computing).

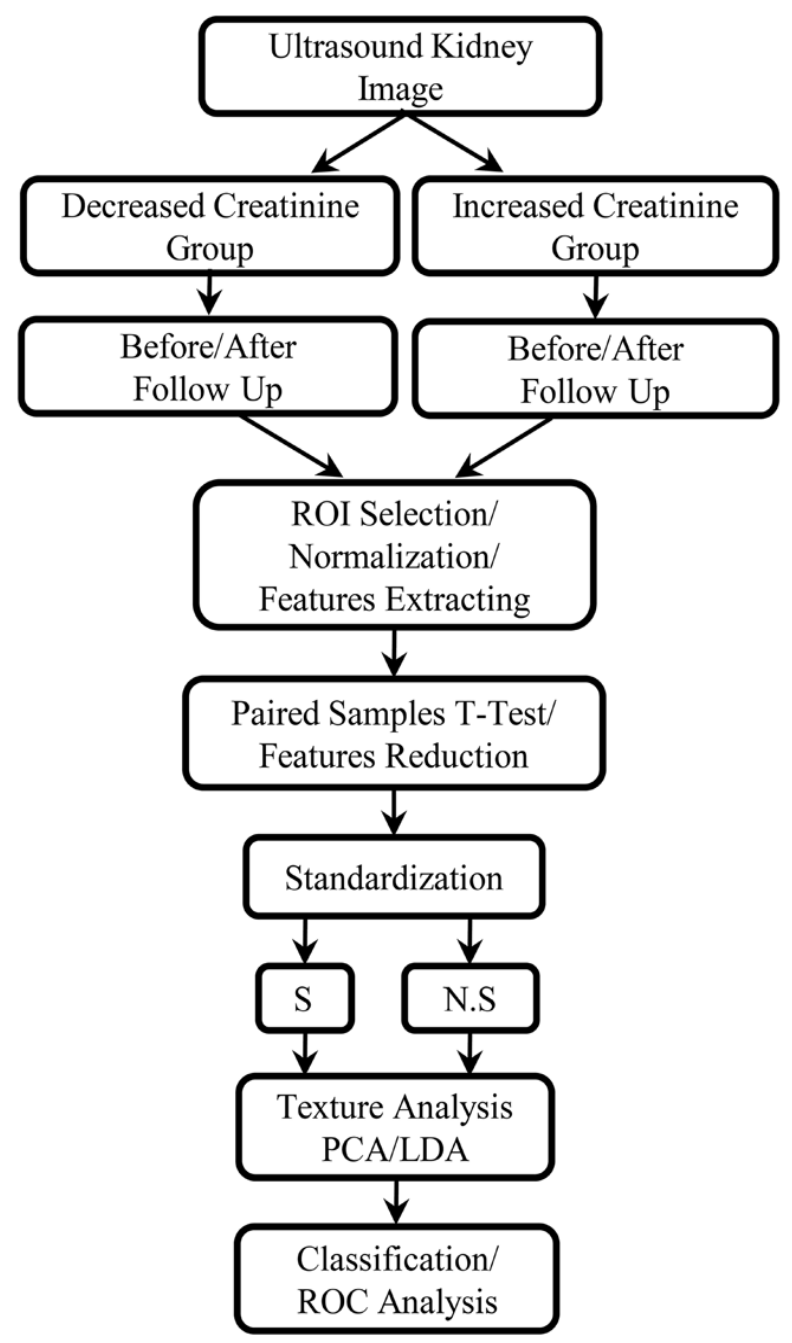

Figure 1: Overview of computer-aided diagnosis (CAD) process in the ultrasound kidney images

\section{Results}

\section{Demographic Data of Patients}

40 kidney allograft recipients (28 male, 12 female) aged $38.9 \pm 15.02$ years (mean age \pm standard deviation) were included in this study. Characteristics of the patients are summarized in Table 1 and Figure 2. Among the 40 patients evaluated in the study, $\mathrm{sCr}$ level increased and decreased in 23 patients (increased group) and 17 patients (decreased group), respectively (11 male, 6 female). The means of $\mathrm{sCr}$ levels for the decreased group before and after followup were $3.84 \pm 1.52$ and $2.78 \pm 1.2$, respectively $(\mathrm{P}=0.007)$ and for increased group were $2.17 \pm$ 0.94 and $4.39 \pm 2.12$, respectively $(\mathrm{P}=0.001)$.

\section{Correlation between Kidney Func- tion and Sonographic Texture Fea- tures}

Among the RLM parameters, most indicated a negative correlation with kidney function in all three normalization schemes for the decreased group. The highest correlation coefficients for default, 3sigma and 1\%-99\% are 135 Dgr_GLNU $(\mathrm{r}=-0.889, \mathrm{P}=0.005), 135$ Dgr_SRE $(\mathrm{r}=0.898, \mathrm{P}<0.001)$ and Horz LRE $(\mathrm{r}=-0.879, \mathrm{P}=0.001)$, respectively (Table 2 ). For the increased group, most significant texture features have significant correlation with $\mathrm{sCr}$ (both positive as well as negative) in all three normalization schemes. The highest correlation coefficients for default, 3sigma and 1\%-99\% are Vert_RLNU ( $\mathrm{r}=0.832, \mathrm{P}=$ 0.038), Vert_RLNU $(\overline{\mathrm{r}}=-0.711, \mathrm{P}=0.004)$ and 135 Dgr_GLNU $(\mathrm{r}=0.765, \mathrm{P}=0.016)$, respectively (Table 3 ).

\section{Texture Classification}

The significant texture features (driven from paired-samples t-test) were implemented for MPTA. The diagnostic performance of the MPTA method for classifying and making a comparison between before and at follow-up in the decreased group is shown in Table 4. According to the normalization role, in 3sig- 
Table 1: Demographic characteristics and laboratory data of patients before and at follow-up.

\begin{tabular}{|c|c|c|c|c|c|c|c|c|c|c|c|c|}
\hline \multicolumn{13}{|c|}{ Increased Group } \\
\hline & \multirow[t]{2}{*}{$\begin{array}{c}\mathrm{BMI} \\
\left(\mathrm{Kg} / \mathrm{m}^{2}\right)\end{array}$} & \multirow[t]{2}{*}{$\begin{array}{l}\text { Obesity } \\
\text { Class }\end{array}$} & \multicolumn{2}{|c|}{$\begin{array}{l}\text { Gender Before } \\
\text { Follow-Up }\end{array}$} & \multirow[t]{2}{*}{ Total } & \multirow[t]{2}{*}{$\begin{array}{c}\text { Age } \\
(\text { Mean } \pm S D)\end{array}$} & \multirow[t]{2}{*}{$\begin{array}{l}\text { Creatinine } \\
\text { (Mean } \pm S D)\end{array}$} & \multicolumn{2}{|c|}{$\begin{array}{c}\text { Gender after } 3 \\
\text { years Follow- } \\
\text { Up }\end{array}$} & \multirow[t]{2}{*}{ Total } & \multirow[t]{2}{*}{$\begin{array}{c}\text { Age } \\
(\text { Mean } \pm S D)\end{array}$} & \multirow[t]{2}{*}{$\begin{array}{l}\text { Creatinine } \\
\text { (Mean } \pm S D)\end{array}$} \\
\hline & & & Male & Female & & & & Male & Female & & & \\
\hline $\begin{array}{l}\text { Under- } \\
\text { weight }\end{array}$ & $<18.5$ & & 1 & - & 1 & 26 & 1.25 & 1 & - & 1 & 26 & 3.8 \\
\hline Normal & $\begin{array}{l}18.5- \\
24.9\end{array}$ & & 14 & 4 & 18 & $40.84 \pm 14.63$ & $2.29 \pm 1.01$ & 15 & 3 & 18 & $33.09 \pm 11.82$ & $3.88 \pm 1.86$ \\
\hline $\begin{array}{l}\text { Over- } \\
\text { weight }\end{array}$ & $\begin{array}{c}25.0- \\
29.9\end{array}$ & & 2 & 1 & 3 & $42.67 \pm 22.48$ & $1.68 \pm 0.11$ & 1 & 2 & 3 & $49.2 \pm 11.77$ & $7.32 \pm 1.74$ \\
\hline \multirow{2}{*}{ Obesity } & $\begin{array}{c}30.0- \\
34.9\end{array}$ & I & - & 1 & 1 & 51 & 2.38 & - & 1 & 1 & 51 & 5.5 \\
\hline & $\begin{array}{c}35.0- \\
39.9\end{array}$ & $\|$ & - & - & - & - & - & - & - & - & - & - \\
\hline $\begin{array}{l}\text { Extreme } \\
\text { Obesity }\end{array}$ & $40.0+$ & III & - & - & - & - & - & - & - & - & - & - \\
\hline
\end{tabular}

\begin{tabular}{|c|c|c|c|c|c|c|c|c|c|c|c|c|}
\hline \multicolumn{13}{|c|}{ Decreased Group } \\
\hline $\begin{array}{l}\text { Under- } \\
\text { weight }\end{array}$ & $<18.5$ & & - & - & - & - & - & 2 & - & 2 & $23 \pm 6$ & $2.6 \pm 0.5$ \\
\hline Normal & $\begin{array}{c}18.5- \\
24.9\end{array}$ & & 11 & 3 & 14 & $34.85 \pm 14.86$ & $3.85 \pm 1.57$ & 8 & 3 & 11 & $34.64 \pm 13.67$ & $2.98 \pm 1.37$ \\
\hline $\begin{array}{l}\text { Over- } \\
\text { weight }\end{array}$ & $\begin{array}{c}25.0- \\
29.9\end{array}$ & & - & 1 & 1 & 27 & 5.2 & 1 & 2 & 3 & $46.67 \pm 14.38$ & $2.5 \pm 0.37$ \\
\hline \multirow{2}{*}{ Obesity } & $\begin{array}{c}30.0- \\
34.9\end{array}$ & । & - & 2 & 2 & $50.5 \pm 1.5$ & 2.38 & - & 1 & 1 & 49 & 1.63 \\
\hline & $\begin{array}{c}35.0- \\
39.9\end{array}$ & $\|$ & - & - & - & - & - & - & - & - & - & - \\
\hline $\begin{array}{l}\text { Extreme } \\
\text { Obesity }\end{array}$ & $40.0+$ & III & - & - & - & - & - & - & - & - & - & - \\
\hline
\end{tabular}

$\mathrm{BMI}=$ Body mass index

ma, RLM features represented the highest performance in terms of sensitivity, specificity, accuracy, PPV and NPV of $100 \%$ by LDA. Moreover, classification tasks performed by S.PCA yielded a high discrimination performance in which sensitivity, specificity, accuracy, PPV, and NPV were 94.12\%, 88.23\%, $91.18 \%, 88.89 \%$, and $93.75 \%$ respectively.

The diagnostic performance of the MPTA method is shown in Table 5. The best result was achieved in 3sigma, features analyzed by LDA with a $95.65 \%$ sensitivity, $91.30 \%$ specificity, 93.47\% accuracy, 91.67\% PPV and 95.45\% NPV. While S.PCA indicated high potential with $78.26 \%$ sensitivity, specificity, accuracy, PPV and NPV.

ROC Analysis

Distribution data under LDA shows that LDA was more powerful in distinguishing 


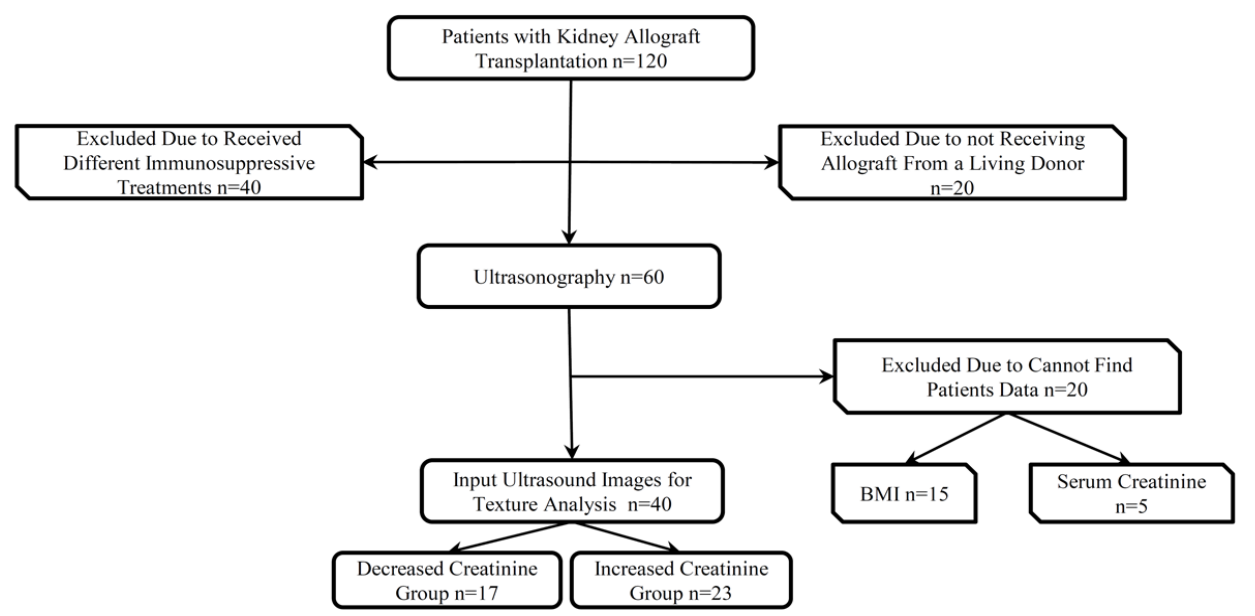

Figure 2: Diagram to report flow of participants through the study.

between before and at follow-up for both the increased and decreased groups (Figure 3). Discriminatory power for the RLM features was examined by constructing ROC curves. Figures 4, 5 and 6 show ROC curves of LDA and PCA for the proposed TA. They were plotted on the same graph for each normalization and patient group (decreased and increased) to make a comparison of discriminating power for classification of patients before and at follow-up. In general, LDA had an advantage over PCA in each normalization and group according to a greater $A_{z}$ value. In classification of the decreased group, the best performance is produced in 3sigma normalization with an $A_{z}$ value of 1. Moreover, it was illustrated, in classification of the increased group, LDA showed the best performance in 3sigma normalization, with an $\mathrm{A}_{\mathrm{z}}$ of 0.974 which corresponding to $95.65 \%$ sensitivity, $91.30 \%$ specificity, 93.47\% accuracy, 91.67\% PPV, and $95.45 \% \mathrm{NPV}$.

\section{Discussion}

The main aim of this study is to assess the potential of texture features in order to monitor kidney function among following up the transplantation recipients using ultrasound imaging.

In all conditions, feature standardization has just an effect on PCA and it leads to improvement in performance. The highest effect regarding to $A_{z}$ value was in 1\%-99\% normalization for both increased group (NS. PCA vs. S.PCA: 0.743 vs. 0.830 ) and decreased one (NS. PCA vs. S.PCA: 0.505 vs. 0.841) (Figure $6)$. It has not had any impacts on LDA. Tables 4 and 5 indicate that normalization reflected improvement on performance and the best performance was obtained by the 3sigma normalization with LDA for both decreased and increased groups with $\mathrm{A}_{\mathrm{z}}$ value of 1 and 0.974, respectively. According to the RLM features, as the $\mathrm{sCr}$ level increases, the non-uniformity of pixel intensities in the ROI also increases.

Over the past a few decades, initial assessments, routine follow-ups, and monitoring of a kidney transplant have been possible via ultrasonography as the primary imaging technique [18]. Moreover, kidney evaluation has been plausible through advanced ultrasonography techniques, including color Doppler and elastographic imaging. Any information on tissue stiffness is included in the elastographic features. Additionally, tissue deformation resulted from compression can be detected through ultrasound elastography. kidney allograft loss can induce fibrosis and tubular atrophy in transplant recipients [19].

Many studies have been carried out to as- 
Table 2: Correlations between serum creatinine $(\mathrm{s} \mathrm{Cr}$ ) level and run-length matrix feature in three normalization schemes for decreased group before and at follow-up.

\begin{tabular}{|c|c|c|c|c|}
\hline Normalization & Texture Feature & $\begin{array}{c}\text { Correlation Coefficient } \\
\text { (P-value) }\end{array}$ & $\begin{array}{l}\text { Paired-Samples } \\
\text { T Test (P-value) }\end{array}$ & ${ }^{*} A_{z}$ value \\
\hline \multirow{5}{*}{ Default } & Horz_GLNU & $-0.881(<0.001)$ & 0.003 & $0.657(0.470,0.845)$ \\
\hline & Vert_GLNU & $-0.894(<0.001)$ & 0.003 & $0.671(0.486,0.856)$ \\
\hline & Vert_RLNU & $-0.843(<0.001)$ & 0.043 & $0.616(0.425,0.807)$ \\
\hline & 45Dgr_GLNU & $-0.886(<0.001)$ & 0.004 & $0.661(0.473,0.848)$ \\
\hline & 135Dgr_GLNU & $-0.889(<0.001)$ & 0.005 & $0.664(0.478,0.851)$ \\
\hline \multirow{18}{*}{ 3Sigma } & Horz_RLNU & $0.693(<0.001)$ & 0.003 & $0.882(0.770,0.995)$ \\
\hline & Horz_GLNU & $-0.823(<0.001)$ & $<0.001$ & $0.834(0.700,0.967)$ \\
\hline & Horz_LRE & $-0.815(<0.001)$ & $<0.001$ & $0.678(0.479,0.877)$ \\
\hline & Horz_SRE & $0.828(<0.001)$ & $<0.001$ & $0.699(0.508,0.890)$ \\
\hline & Horz_Fraction & $0.824(<0.001)$ & $<0.001$ & $0.692(0.498,0.886)$ \\
\hline & Vert_RLNU & $-0.571(<0.001)$ & 0.037 & $0.619(0.398,0.841)$ \\
\hline & Vert_GLNU & $-0.829(<0.001)$ & $<0.001$ & $0.761(0.595,0.927)$ \\
\hline & Vert_LRE & $-0.762(<0.001)$ & $<0.001$ & $0.730(0.548,0.913)$ \\
\hline & Vert_SRE & $0.760(<0.001)$ & $<0.001$ & $0.751(0.578,0.923)$ \\
\hline & Vert_Fraction & $0.756(<0.001)$ & $<0.001$ & $0.744(0.568,0.920)$ \\
\hline & 45Dgr_GLNU & $-0.826(<0.001)$ & $<0.001$ & $0.758(0.590,0.926)$ \\
\hline & 45Dgr_LRE & $-0.875(<0.001)$ & $<0.001$ & $0.692(0.498,0.886)$ \\
\hline & 45Dgr_SRE & $0.875(<0.001)$ & $<0.001$ & $0.723(0.542,0.905)$ \\
\hline & 45Dgr_Fraction & $0.881(<0.001)$ & $<0.001$ & $0.702(0.513,0.892)$ \\
\hline & 135Dgr_GLNU & $-0.829(<0.001)$ & $<0.001$ & $0.761(0.595,0.927)$ \\
\hline & 135Dgr_LRE & $-0.878(<0.001)$ & $<0.001$ & $0.706(0.518,0.894)$ \\
\hline & 135Dgr_SRE & $0.898(<0.001)$ & $<0.001$ & $0.706(0.517,0.894)$ \\
\hline & 135Dgr_Fraction & $0.878(<0.001)$ & $<0.001$ & $0.713(0.527,0.898)$ \\
\hline \multirow{7}{*}{$1 \%-99 \%$} & Horz_GLNU & $-0.792(<0.001)$ & $<0.001$ & $0.727(0.553,0.900)$ \\
\hline & Horz_LRE & $-0.879(<0.001)$ & 0.001 & $0.626(0.412,0.841)$ \\
\hline & Vert_GLNU & $-0.796(<0.001)$ & $<0.001$ & $0.709(0.529,0.889)$ \\
\hline & Vert_LRE & $-0.569(<0.001)$ & $<0.001$ & $0.592(0.381,0.803)$ \\
\hline & Vert_SRE & $0.635(<0.001)$ & $<0.001$ & $0.595(0.389,0.801)$ \\
\hline & 45Dgr_GLNU & $-0.749(<0.001)$ & $<0.001$ & $0.713(0.534,0.892)$ \\
\hline & 135Dgr_GLNU & $-0.797(<0.001)$ & $<0.001$ & $0.713(0.534,0.892)$ \\
\hline
\end{tabular}

\footnotetext{
* A value was obtained with $95 \%$ confidence intervals.
} 
Abbasian Ardakani A. et al

Table 3: Correlations between serum creatinine $(\mathrm{sCr})$ level and run-length matrix feature in three normalization schemes for increased group before and at follow-up.

\begin{tabular}{|c|c|c|c|c|}
\hline $\begin{array}{c}\text { Texture Feature } \\
\text { Group }\end{array}$ & Texture Feature & $\begin{array}{c}\text { Correlation Coefficient } \\
\text { (P-value) }\end{array}$ & $\begin{array}{c}\text { Paired-Samples T Test } \\
\text { (P-value) }\end{array}$ & ${ }^{*} A_{z}$ value \\
\hline \multirow{8}{*}{ Default } & Horz_RLNU & $0.364(0.017)$ & 0.001 & $0.779(0.643,0.914)$ \\
\hline & Horz_GLNU & $-0.580(0.027)$ & $<0.001$ & $0.853(0.736,0.969)$ \\
\hline & Vert_RLNU & $0.832(0.038)$ & $<0.001$ & $0.932(0.864,1.000)$ \\
\hline & Vert_GLNU & $-0.209(0.004)$ & $<0.001$ & $0.866(0.754,0.978)$ \\
\hline & 45Dgr_RLNU & $0.500(0.014)$ & $<0.001$ & $0.915(0.838,0.992)$ \\
\hline & 45Dgr_GLNU & $-0.182(0.022)$ & $<0.001$ & $0.851(0.729,0.972)$ \\
\hline & 135Dgr_RLNU & $0.550(0.001)$ & $<0.001$ & $0.926(0.854,0.998)$ \\
\hline & 135Dgr_GLNU & $-0.372(0.015)$ & $<0.001$ & $0.856(0.739,0.973)$ \\
\hline \multirow{13}{*}{ 3Sigma } & Horz_RLNU & $-0.192(0.002)$ & $<0.001$ & $0.875(0.776,0.974)$ \\
\hline & Horz_GLNU & $0.316(0.038)$ & $<0.001$ & $0.877(0.777,0.977)$ \\
\hline & Vert_RLNU & $-0.711(0.004)$ & $<0.001$ & $0.924(0.852,0.997)$ \\
\hline & Vert_GLNU & $0.630(0.003)$ & $<0.001$ & $0.879(0.783,0.975)$ \\
\hline & Vert_LRE & $0.670(<0.001)$ & 0.049 & $0.626(0.459,0.792)$ \\
\hline & Vert_Fraction & $-0.676(<0.001)$ & 0.040 & $0.631(0.465,0.798)$ \\
\hline & 45Dgr_RLNU & $-0.243(0.018)$ & $<0.001$ & $0.909(0.829,0.990)$ \\
\hline & 45Dgr_GLNU & $0.545(0.042)$ & $<0.001$ & $0.870(0.769,0.970)$ \\
\hline & 135Dgr_RLNU & $-0.579(0.005)$ & $<0.001$ & $0.921(0.846,0.995)$ \\
\hline & 135Dgr_GLNU & $0.513(0.009)$ & $<0.001$ & $0.871(0.772,0.971)$ \\
\hline & 135Dgr_LRE & $0.696(<0.001)$ & 0.028 & $0.635(0.469,0.802)$ \\
\hline & 135Dgr_SRE & $-0.697(<0.001)$ & 0.035 & $0.626(0.456,0.795)$ \\
\hline & 135Dgr_Fraction & $-0.699(<0.001)$ & 0.027 & $0.629(0.461,0.798)$ \\
\hline \multirow{7}{*}{$1 \%-99 \%$} & Horz_GLNU & $-0.693(0.012)$ & $<0.001$ & $0.896(0.804,0.988)$ \\
\hline & Horz_LRE & $0.287(0.035)$ & 0.001 & $0.709(0.557,0.861)$ \\
\hline & Vert_GLNU & $0.744(0.013)$ & $<0.001$ & $0.900(0.808,0.992)$ \\
\hline & Vert_LRE & $0.456(0.001)$ & $<0.001$ & $0.531(0.359,0.703)$ \\
\hline & Vert_SRE & $-0.460(0.001)$ & $<0.001$ & $0.507(0.337,0.676)$ \\
\hline & 45Dgr_GLNU & $0.388(0.010)$ & $<0.001$ & $0.892(0.794,0.990)$ \\
\hline & 135Dgr_GLNU & $0.765(0.016)$ & $<0.001$ & $0.900(0.805,0.994)$ \\
\hline
\end{tabular}

* $A_{z}$ value was obtained with $95 \%$ confidence intervals.

sess correlation between kidney function and sonographic finding. In this regard, it has been shown that there is no agreement on the im- pact of pathology changes on Doppler and elastography findings. Some studies reported correlation between pathological changes and 
Kidney Function Assessment Using Texture Analysis

Table 4: Correlations between serum creatinine $(\mathrm{sCr})$ level and run-length matrix feature in three normalization schemes for decreased group before and at follow-up.

\begin{tabular}{|c|c|c|c|c|c|c|c|c|}
\hline Normalization & $\begin{array}{l}\text { Method of feature } \\
\text { reduction }\end{array}$ & $\begin{array}{l}\text { a SEN } \\
\text { (\%) }\end{array}$ & $\begin{array}{l}\text { a SPC } \\
(\%)\end{array}$ & $\begin{array}{c}\text { ACC } \\
(\%)\end{array}$ & $\begin{array}{l}\text { a PPV } \\
\text { (\%) }\end{array}$ & $\begin{array}{l}\text { a NPV } \\
\text { (\%) }\end{array}$ & ${ }^{\mathrm{a}} \mathrm{A}_{\mathrm{z}}$ value & $\begin{array}{l}\text { Correct classifi- } \\
\text { cation }\end{array}$ \\
\hline \multirow{4}{*}{ Default } & NS. PCA & $\begin{array}{c}47.06 \\
(32.03, \\
77.08)\end{array}$ & $\begin{array}{c}41.18 \\
(34.83, \\
87.14)\end{array}$ & 44.12 & $\begin{array}{c}44.43 \\
(07.66 \\
81.2)\end{array}$ & $\begin{array}{r}43.75 \\
(03.61, \\
83.89)\end{array}$ & $\begin{array}{c}0.484 \\
(0.282 \\
0.686)\end{array}$ & $15 / 34$ [44.18\%] \\
\hline & S. PCA & $\begin{array}{c}58.82 \\
(41.18 \\
82.35)\end{array}$ & $\begin{array}{c}64.70 \\
(62.47 \\
8154)\end{array}$ & 61.76 & $\begin{array}{r}62.50 \\
(39.55 \\
85.45)\end{array}$ & $\begin{array}{r}61.10 \\
(41.60 \\
80.60)\end{array}$ & $\begin{array}{c}0.657 \\
(0.465 \\
0.849)\end{array}$ & 21/34 [61.76\%] \\
\hline & NS. LDA & $\begin{array}{c}82.35 \\
(64.71 \\
100)\end{array}$ & $\begin{array}{c}88.23 \\
(67.50 \\
100)\end{array}$ & 85.29 & $\begin{array}{c}87.50 \\
(74.49 \\
100)\end{array}$ & $\begin{array}{l}83.30 \\
(66.73 \\
99.87)\end{array}$ & $\begin{array}{c}0.927 \\
(0.840 \\
1.000)\end{array}$ & 29/34 [85.29\%] \\
\hline & S. LDA & $\begin{array}{c}82.35 \\
(64.71 \\
100)\end{array}$ & $\begin{array}{c}88.23 \\
(67.50 \\
1.00)\end{array}$ & 85.29 & $\begin{array}{c}87.50 \\
(74.49 \\
100)\end{array}$ & $\begin{array}{l}83.30 \\
(66.73 \\
99.87)\end{array}$ & $\begin{array}{c}0.927 \\
(0.840 \\
1.000)\end{array}$ & 29/34 [85.29\%] \\
\hline \multirow{4}{*}{ 3sigma } & NS. PCA & $\begin{array}{c}88.23 \\
(47.06 \\
100)\end{array}$ & $\begin{array}{c}88.23 \\
(67.50 \\
100)\end{array}$ & 88.23 & $\begin{array}{c}88.23 \\
(76.16 \\
100)\end{array}$ & $\begin{array}{c}88.23 \\
(63.10 \\
100)\end{array}$ & $\begin{array}{c}0.938 \\
(0.863 \\
1.000)\end{array}$ & 30/34 [88.23\%] \\
\hline & S. PCA & $\begin{array}{c}94.12 \\
(82.35, \\
100)\end{array}$ & $\begin{array}{c}88.23 \\
(76.47 \\
100)\end{array}$ & 91.18 & $\begin{array}{c}88.89 \\
(77.70 \\
100)\end{array}$ & $\begin{array}{c}93.75 \\
(42.25 \\
100)\end{array}$ & $\begin{array}{c}0.962 \\
(0.906 \\
1.000)\end{array}$ & $31 / 34$ [91.18\%] \\
\hline & NS. LDA & 100 & 100 & 100 & 100 & 100 & 1 & $34 / 34$ [100\%] \\
\hline & S. LDA & 100 & 100 & 100 & 100 & 100 & 1 & $34 / 34$ [100\%] \\
\hline \multirow{4}{*}{$1-99 \%$} & NS. PCA & $\begin{array}{r}52.94 \\
(17.65, \\
94.12)\end{array}$ & $\begin{array}{r}41.18 \\
(29.94 \\
74.26)\end{array}$ & 47.06 & $\begin{array}{r}47.37 \\
(10.56, \\
84.18)\end{array}$ & $\begin{array}{r}46.67 \\
(11.09, \\
82.25)\end{array}$ & $\begin{array}{c}0.505 \\
(0.306 \\
0.705)\end{array}$ & $16 / 34$ [47.06\%] \\
\hline & S. PCA & $\begin{array}{r}70.59 \\
(40.88 \\
94.12)\end{array}$ & $\begin{array}{c}88.23 \\
(64.71 \\
100)\end{array}$ & 79.41 & $\begin{array}{c}85.71 \\
(70.56 \\
100)\end{array}$ & $\begin{array}{c}75 \\
(64.82 \\
85.18)\end{array}$ & $\begin{array}{c}0.841 \\
(0.706 \\
0.976)\end{array}$ & $27 / 34$ [79.41\%] \\
\hline & NS. LDA & $\begin{array}{c}94.12 \\
(61.62, \\
100)\end{array}$ & $\begin{array}{c}82.35 \\
(73.38 \\
100)\end{array}$ & 88.23 & $\begin{array}{r}84.21 \\
(76.53 \\
91.89)\end{array}$ & $\begin{array}{c}93.33 \\
(85.81 \\
100)\end{array}$ & $\begin{array}{c}0.934 \\
(0.856 \\
1.000)\end{array}$ & 30/34 [88.23\%] \\
\hline & S. LDA & $\begin{array}{c}94.12 \\
(61.62, \\
100)\end{array}$ & $\begin{array}{c}82.35 \\
(73.38 \\
100)\end{array}$ & 88.23 & $\begin{array}{r}84.21 \\
(76.53 \\
91.89)\end{array}$ & $\begin{array}{c}93.33 \\
(85.81 \\
100)\end{array}$ & $\begin{array}{c}0.934 \\
(0.856 \\
1.000)\end{array}$ & $30 / 34$ [88.23\%] \\
\hline
\end{tabular}

$\mathrm{SEN}=$ sensitivity SPC $=$ specificity; $\mathrm{ACC}=$ accuracy $; \mathrm{PPV}=$ positive predictive value; $\mathrm{NPV}=$ negative predictive value; $\mathrm{A}_{\mathrm{z}}=$ area under ROC curve; a: Numbers in parentheses are $95 \%$ confidence intervals.

resistance index (RI) or kidney stiffness $(\mathrm{KS})$ [20-23] and some others showed the opposite $[24,25]$. Our pervious study showed that sonographic quantitative TA can distinguish reject from non-reject kidney transplant with higher accuracy comparing with RI and KS [26]. In other way, Wybraniec et al., [27] and Komuro et al., [28] indicated that RI correlated with sCr level but Yazici et al., [29] showed opposite. Besides, in the term of ellastography, Peng et al., [30] showed that elastographic findings were correlated with sCr level while 
Table 5: Diagnostic performance of proposed method in three normalization schemes for increased creatinine group.

\begin{tabular}{|c|c|c|c|c|c|c|c|c|}
\hline Normalization & $\begin{array}{l}\text { Method of feature } \\
\text { reduction }\end{array}$ & $\begin{array}{l}{ }^{a} \text { SEN } \\
(\%)\end{array}$ & $\begin{array}{l}{ }^{a} \text { SPC } \\
(\%)\end{array}$ & $\begin{array}{c}\text { ACC } \\
(\%)\end{array}$ & $\begin{array}{l}\text { a PPV } \\
(\%)\end{array}$ & $\begin{array}{l}\text { a NPV } \\
(\%)\end{array}$ & ${ }^{\mathrm{a}} \mathrm{A}_{\mathrm{z}}$ value & $\begin{array}{c}\text { Correct classifi- } \\
\text { cation }\end{array}$ \\
\hline \multirow{4}{*}{ Default } & NS. PCA & $\begin{array}{l}65.22 \\
(48.54 \\
86.36)\end{array}$ & $\begin{array}{c}73.91 \\
(58.01, \\
94.75)\end{array}$ & 69.56 & $\begin{array}{c}71.43 \\
(51.68 \\
91.18)\end{array}$ & $\begin{array}{l}68.00 \\
(57.30 \\
78.70)\end{array}$ & $\begin{array}{c}0.750(0.607 \\
0.894)\end{array}$ & $32 / 46$ [69.56\%] \\
\hline & S. PCA & $\begin{array}{r}69.56 \\
(39.18 \\
87.26)\end{array}$ & $\begin{array}{r}78.26 \\
(61.37 \\
94.89)\end{array}$ & 73.91 & $\begin{array}{r}76.19 \\
(60.18 \\
92.20)\end{array}$ & $\begin{array}{c}72.00 \\
(61.72, \\
82.28)\end{array}$ & $\begin{array}{c}0.798(0.668, \\
0.928)\end{array}$ & $34 / 46$ [73.91\%] \\
\hline & NS. LDA & $\begin{array}{r}86.96 \\
(79.27 \\
94.79)\end{array}$ & $\begin{array}{c}91.30 \\
(87.94 \\
100)\end{array}$ & 89.13 & $\begin{array}{c}90.90 \\
(81.25 \\
100)\end{array}$ & $\begin{array}{r}87.50 \\
(75.49, \\
99.51)\end{array}$ & $\begin{array}{c}0.949(0.893 \\
1.000)\end{array}$ & $41 / 46$ [89.13\%] \\
\hline & S. LDA & $\begin{array}{r}86.96 \\
(79.27 \\
94.79)\end{array}$ & $\begin{array}{c}91.30 \\
(87.94 \\
100)\end{array}$ & 89.13 & $\begin{array}{c}90.90 \\
(81.25 \\
100)\end{array}$ & $\begin{array}{c}87.50 \\
(75.49, \\
99.51)\end{array}$ & $\begin{array}{c}0.949(0.893, \\
1.000)\end{array}$ & $41 / 46$ [89.13\%] \\
\hline \multirow{4}{*}{ 3sigma } & NS. PCA & $\begin{array}{l}78.26 \\
(58.82 \\
97.21)\end{array}$ & $\begin{array}{r}69.56 \\
(35.29 \\
94.12)\end{array}$ & 73.91 & $\begin{array}{c}72 \\
(53.51 \\
90.49)\end{array}$ & $\begin{array}{c}76.19 \\
(54.86 \\
97.52)\end{array}$ & $\begin{array}{c}0.790 \\
(0.65,0.93)\end{array}$ & $34 / 46$ [73.91\%] \\
\hline & S. PCA & $\begin{array}{l}78.26 \\
(63.38 \\
88.96)\end{array}$ & $\begin{array}{r}78.26 \\
(54.18 \\
92.22)\end{array}$ & 78.26 & $\begin{array}{r}78.26 \\
(64.33 \\
92.19)\end{array}$ & $\begin{array}{c}78.26 \\
(63.73, \\
92.79)\end{array}$ & $\begin{array}{c}0.828(0.701 \\
0.955)\end{array}$ & $36 / 46$ [78.26\%] \\
\hline & NS. LDA & $\begin{array}{c}95.65 \\
(76.35 \\
100)\end{array}$ & $\begin{array}{c}91.3 \\
(70.66 \\
100)\end{array}$ & 93.47 & $\begin{array}{c}91.67 \\
(83.22 \\
100)\end{array}$ & $\begin{array}{c}95.45 \\
(56.81, \\
100)\end{array}$ & $\begin{array}{c}0.974(0.936 \\
1.000)\end{array}$ & $43 / 46$ [93.48\%] \\
\hline & NS. LDA & $\begin{array}{c}95.65 \\
(76.35 \\
100)\end{array}$ & $\begin{array}{c}91.3 \\
(70.66 \\
100)\end{array}$ & 93.47 & $\begin{array}{c}91.67 \\
(83.22 \\
100)\end{array}$ & $\begin{array}{c}95.45 \\
(56.81, \\
100)\end{array}$ & $\begin{array}{c}0.974(0.936 \\
1.000)\end{array}$ & $43 / 46[93.48 \%]$ \\
\hline \multirow{4}{*}{$1-99 \%$} & NS. PCA & $\begin{array}{r}65.22 \\
(35.00 \\
82.35)\end{array}$ & $\begin{array}{c}69.56 \\
(26.32 \\
94.12)\end{array}$ & 67.39 & $\begin{array}{r}68.18 \\
(45.52 \\
90.84)\end{array}$ & $\begin{array}{r}66.67 \\
(53.99, \\
79.35)\end{array}$ & $\begin{array}{c}0.743(0.596 \\
0.89)\end{array}$ & $31 / 46$ [67.39\%] \\
\hline & S. PCA & $\begin{array}{c}82.61 \\
(67.50 \\
100)\end{array}$ & $\begin{array}{c}73.91 \\
(70.59 \\
100)\end{array}$ & 78.26 & $\begin{array}{c}76 \\
(61.38 \\
90.62)\end{array}$ & $\begin{array}{c}80.95 \\
(58.43 \\
100)\end{array}$ & $\begin{array}{c}0.830(0.712 \\
0.948)\end{array}$ & $36 / 46$ [78.26\%] \\
\hline & NS. LDA & $\begin{array}{c}91.3 \\
(85.02 \\
100)\end{array}$ & $\begin{array}{c}82.61 \\
(81.21, \\
88.90)\end{array}$ & 86.96 & $\begin{array}{c}84 \\
(76.11 \\
91.89)\end{array}$ & $\begin{array}{c}90.48 \\
(59.51 \\
100)\end{array}$ & $\begin{array}{c}0.934(0.867 \\
1.000)\end{array}$ & $40 / 46$ [86.97\%] \\
\hline & S. LDA & 91.3 & 82.61 & 86.96 & 84 & 90.48 & $\begin{array}{c}0.934(0.867 \\
1.000)\end{array}$ & $40 / 46$ [86.97\%] \\
\hline
\end{tabular}

$\mathrm{SEN}=$ sensitivity SPC $=$ specificity $; \mathrm{ACC}=$ accuracy $; \mathrm{PPV}=$ positive predictive value NPV = negative predictive value; $\mathrm{A}=$ area under ROC curve; a: Numbers in parentheses are $95 \%$ confidence intervals.

in study carried out by Lee et al., no correlation was observed [31]. Further researches are needed, in Doppler and elato-sonography field to reach agreement on findings.

Moreover, our study indicated that texture parameters have higher correlation coefficients with sCr level comparing to both Doppler and elastographic indices. The main results of our previous study [26] reveal that TA can monitor kidney function and correlate with pathologi- 


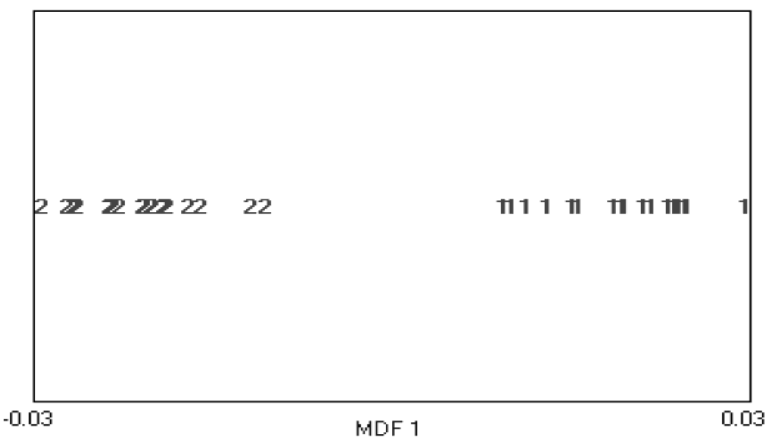

A

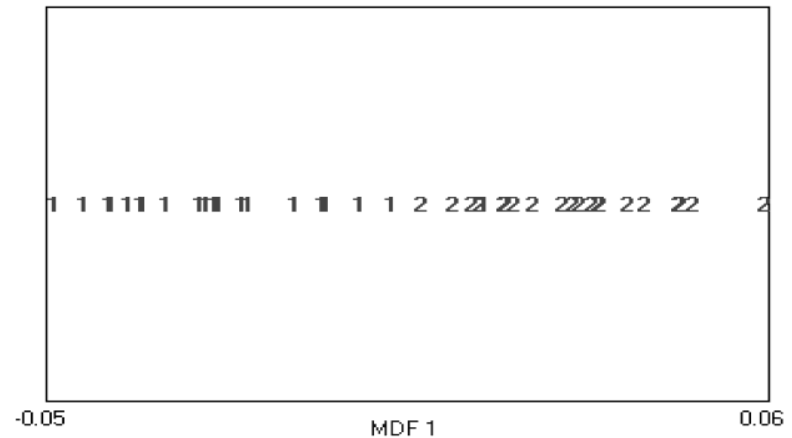

B

Figure 3: Sample distributions for the best results after texture analysis method, linear discriminant analysis (LDA): 3sigma normalization for decreased group (A) and for increased group (B). Most discriminating features (MDF); " 1 " and " 2 " represent patients before and after follow-up, respectively.

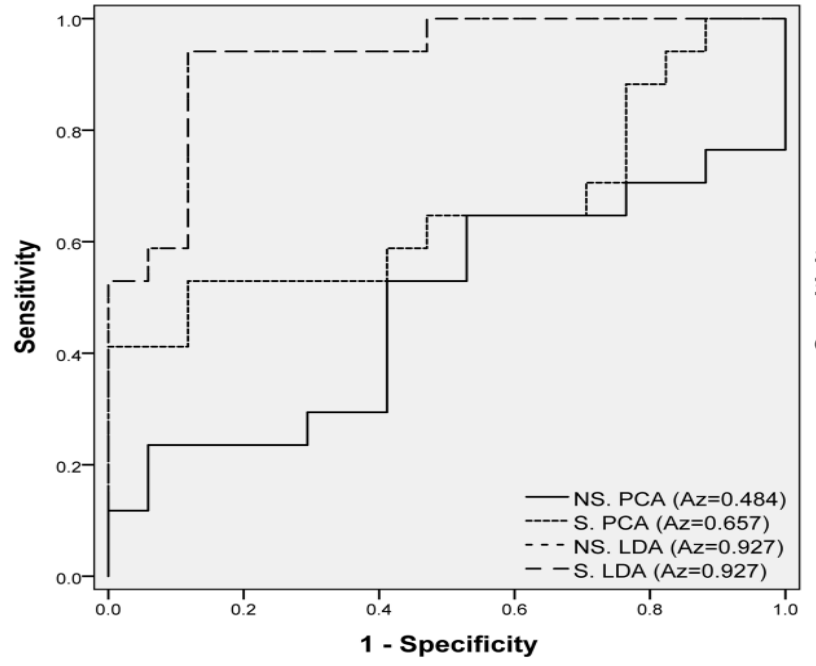

A

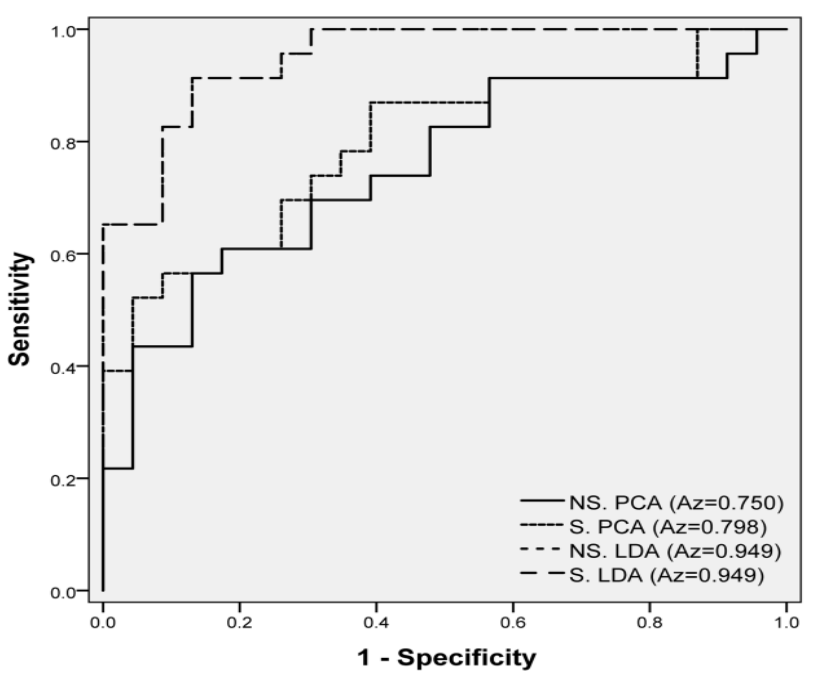

B

Figure 4: The diagrams of the receiver operating characteristic (ROC) curve for each texture analysis method in default: (A) decreased group; (B) increased group.

cal results, while the present study indicates that TA can evaluate $\mathrm{sCr}$ level of patient after 3years follow-up since allograft transplantation. Our previous results were obtained in normal conditions, while in the present study, owing to the lack of biopsy results, we have to increase the diagnostic accuracy by using normalization and standardization options on texture parameters. In this regard, the best re- sults were achieved on 3sigma normalization. These texture parameters are quantitatively measured by computer software and it has reproducibility capability. But Doppler and elasto-sonography techniques are operated dependently and also can be affected by factors such as skin-allograft distance, peri or intra renal fluid, gender, age and sonicated direction $[25,32]$. 


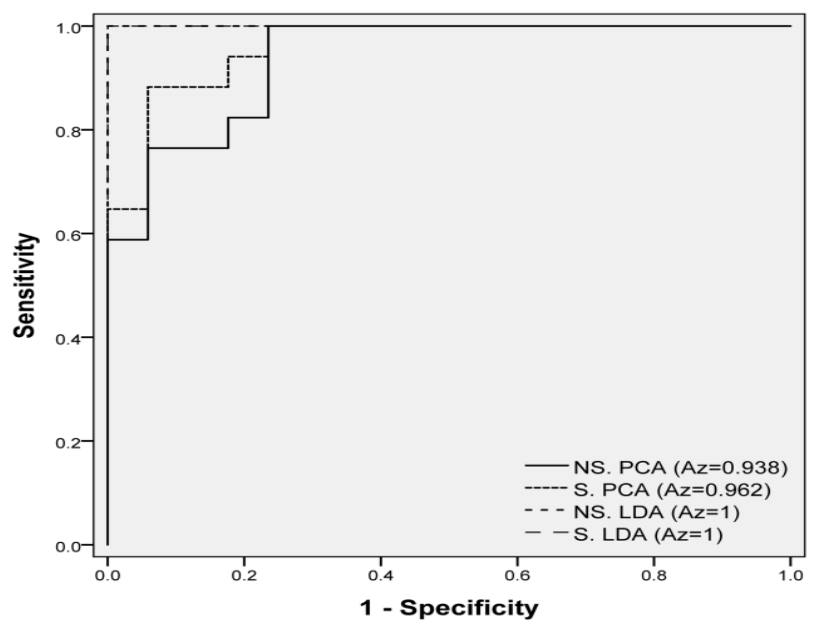

A

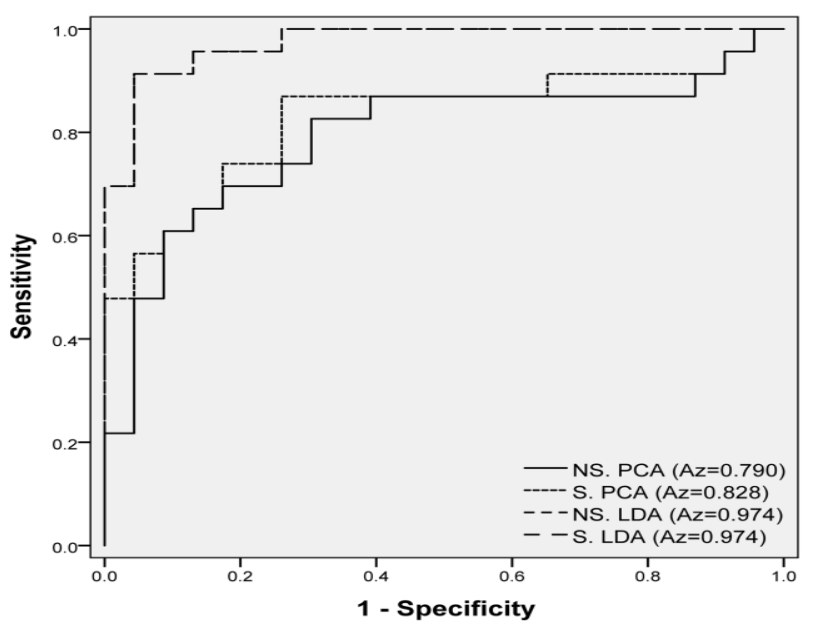

B

Figure 5: The diagrams of the receiver operating characteristic (ROC) curve for each texture analysis method in 3sigma normalization: (A) decreased group; (B) increased group.

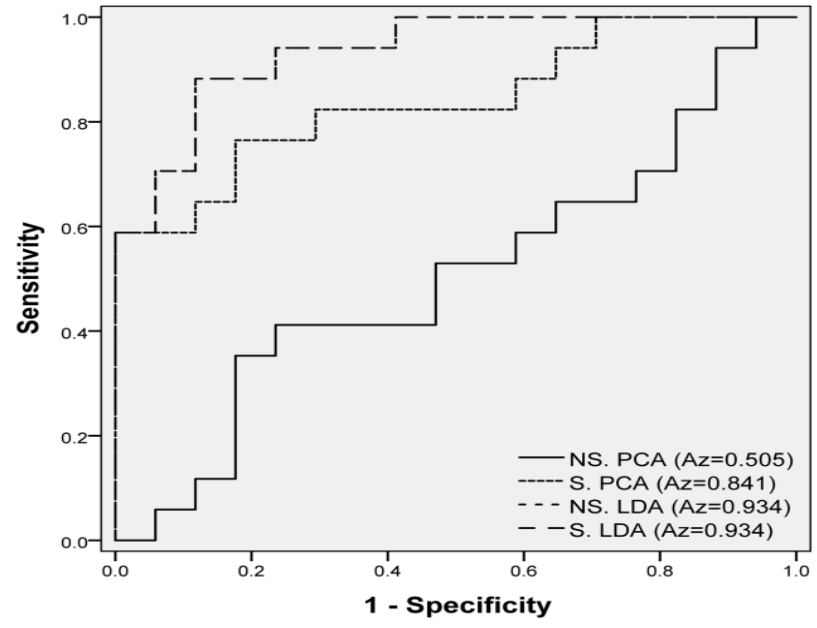

A

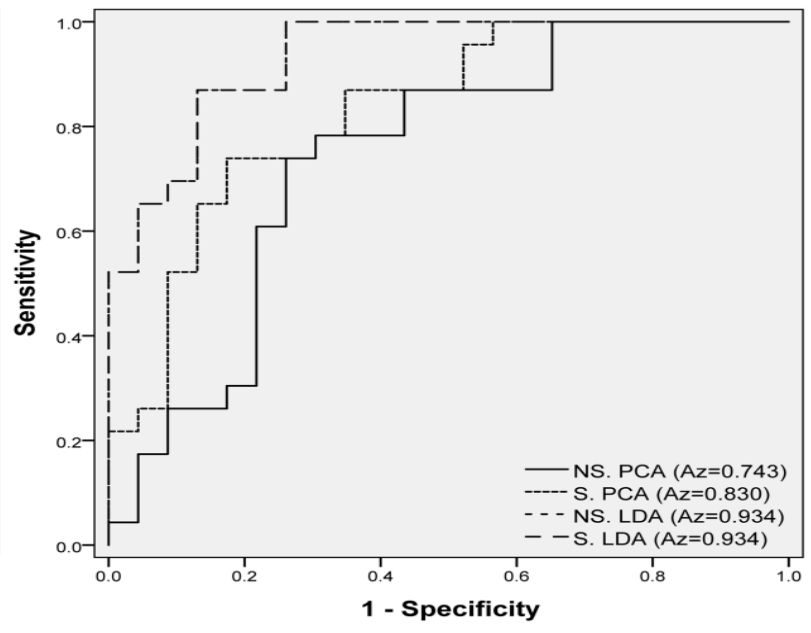

B

Figure 6: The diagrams of the receiver operating characteristic (ROC) curve for each texture analysis method in 1-99\% normalization: (A) decreased group; (B) increased group.

Kidney function monitoring is one of the most critical factors to improve initial diagnosis accuracy after transplantation. Our results indicated that TA can monitor kidney function after transplantation. Further studies with larger dataset should be performed to confirm this result. Moreover, sCr levels were used to assess kidney function and correlate with texture parameters. Cystatin $\mathrm{C}$ has an advantage over $\mathrm{sCr}$ and can reflect true glomerular filtration rate $[33,34]$. Besides sex, muscle mass, age and medication can influence sCr level [35, 36]. Further studies require to be performed with respect to cystatin $\mathrm{C}$ to assess kidney function.

Kidney images were compared with sCr lev- 
Kidney Function Assessment Using Texture Analysis

els and the reference for further investigations. Since the current study was based on image texture analysis and our reference was $\mathrm{sCr}$, other kidney parameters were not considered.

\section{Conclusion}

In this study, we propose a new approach of texture features ability for predicting renal function. This method can be used to help physicians monitor kidney dysfunction and identify candidate patients for biopsy after transplantation. It can be used as an axillary technique with Doppler and elastography sonography to improve understandings of conventional ultrasound imaging.

\section{Conflict of Interest}

None

\section{References}

1. Centers for Disease Control and Prevention. National Chronic Kidney Disease Fact Sheet: general information and national estimates on chronic kidney disease in the United States, 2010. Atlanta, Department of Health and Human Services, Centers for Disease Control and Prevention; 2010.

2. Matas AJ, Smith JM, Skeans MA, Lamb KE, Gustafson SK, Samana CJ, et al. OPTN/SRTR 2011 Annual Data Report: kidney. Am J Transplant. 2013;13 Suppl 1:11-46. doi: 10.1111/ajt.12019. PubMed PMID: 23237695.

3. Forbes JM, Hewitson TD, Becker GJ, Jones CL. Ischemic acute renal failure: long-term histology of cell and matrix changes in the rat. Kidney Int. 2000;57:2375-85. doi: 10.1046/j.15231755.2000.00097.x.

4. Simmonds MJ. Using genetic variation to predict and extend long-term kidney transplant function. Transplantation. 2015;99:2038-48. doi: 10.1097/ tp.0000000000000836.

5. Merhi B, Bayliss G, Gohh RY. Role for urinary biomarkers in diagnosis of acute rejection in the transplanted kidney. World J Transplant. 2015;5:25160. doi: 10.5500/wjt.v5.i4.251. PubMed PMID: 26722652. PubMed PMCID: PMC4689935.

6. Wymer DC. Imaging the Chronic Kidney Disease Patient: Clinical Approaches, Utility and Complications. Chronic Renal Disease. 2015:890-6. doi: 10.1016/b978-0-12-411602-3.00075-5.

7. Castellano G, Bonilha L, Li LM, Cendes F. Tex- ture analysis of medical images. Clin Radiol. 2004;59:1061-9. doi: 10.1016/j.crad.2004.07.008. PubMed PMID: 15556588.

8. Abbasian Ardakani A, Gharbali A, Saniei Y, Mosarrezaii A, Nazarbaghi S. Application of Texture Analysis in Diagnosis of Multiple Sclerosis by Magnetic Resonance Imaging. Glob J Health Sci. 2015;7:6878. doi: 10.5539/gjhs.v7n6p68. PubMed PMID: 26153164. PubMed PMCID: PMC4803872.

9. Raja KB, Madheswaran M, Thyagarajah K. Texture pattern analysis of kidney tissues for disorder identification and classification using dominant Gabor wavelet. Machine Vision and Applications. 2010;21:287-300.

10. Hafizah WM, Supriyanto E, Yunus J, editors. Feature extraction of kidney ultrasound images based on intensity histogram and gray level cooccurrence matrix. Sixth Asia Modelling Symposium; Bali, Indonesia: IEEE; 2012. doi: 10.1109/ ams.2012.47.

11. Attia MW, Abou-Chadi F, Moustafa HE-D, Mekky $N$. Classification of ultrasound kidney images using PCA and neural networks. International Journal of Advanced Computer Science and Applications. 2015;6:53-7. doi: 10.14569/IJACSA.2015.060407.

12. Subramanya M, Kumar V, Mukherjee S, Saini M. SVM-based CAC system for B-mode kidney ultrasound images. J Digit Imaging. 2015;28:448-58. doi: 10.1007/s10278-014-9754-4.

13. Pujari RM, Hajare VD, editors. Analysis of ultrasound images for identification of Chronic Kidney Disease stages. First International Conference on Networks \& Soft Computing (ICNSC2014); Guntur, India: IEEE. 2014. p. 19-20. doi: 10.1109/ cnsc.2014.6906704.

14. Chen C-J, Pai T-W, Fujita H, Lee C-H, Chen Y-T, Chen K-S, editors. Stage diagnosis for Chronic Kidney Disease based on ultrasonography. 11th International Conference on Fuzzy Systems and Knowledge Discovery (FSKD); Xiamen, China: IEEE; 2014. doi: 0.1109/FSKD.2014.6980889.

15. Raja KB, Madheswaran M, Thyagarajah K. Ultrasound kidney image analysis for computerized disorder identification and classification using content descriptive power spectral features. J Med Syst. 2007;31:307-17. doi: 10.1007/s10916-0079068-x. PubMed PMID: 17918683.

16. Raja KB, Madheswaran M, Thyagarajah K. A hybrid fuzzy-neural system for computer-aided diagnosis of ultrasound kidney images using prominent features. J Med Syst. 2008;32:65-83. doi: 10.1007/ s10916-007-9109-5. 
17. National Heart L, Institute B. Classification of overweight and obesity by BMI, waist circumference, and associated disease risks. Retrieved March. 2015;12:2015.

18. Parthipun A, Pilcher J. Renal transplant assessment: sonographic imaging. Ultrasound Clinics. 2010;5:379-99. doi: 10.1016/j.cult.2010.08.004.

19. Fletcher JT, Nankivell BJ, Alexander SI. Chronic allograft nephropathy. Pediatr Nephrol. 2009;24:1465-71.

20. Peters B, Stegmayr B, Mölne J, Haux SB, Hadimeri H. High Resistive Index in Transplant Kidneys Is a Possible Predictor for Biopsy Complications. Transplant Proc. 2016;48:2714-17. doi: 10.1016/j. transproceed.2016.07.016. PubMed PMID: 27788806.

21. Liu J, Blumfield A, Trivedi M, Mishra N, Mohammed 0, Lin J, et al. Does Assessing Preimplantation Kidney Biopsy and Pump Resistive Index Values Predict Kidney Allograft Survival? Transplantation. 2018;102:S32. doi: 10.1097/01. tp.0000542583.11359.05.

22. Yang J, Wang F, Pan W, Ruan L, Ai H. Correlation between ultrasound elastography parameters and renal function after kidney transplantation. Int $J$ Clin Exp Med. 2017;10:3211-7.

23. Ghonge NP, Mohan M, Kashyap V, Jasuja S. Renal Allograft Dysfunction: Evaluation with Shear-wave Sonoelastography. Radiology. 2018;288:146-52. doi: 10.1148/radiol.2018170577. PubMed PMID: 29634441.

24. Gao J, Rubin JM, Weitzel W, Lee J, Dadhania D, Kapur S, et al. Comparison of Ultrasound Corticomedullary Strain with Doppler Parameters in Assessment of Renal Allograft Interstitial Fibrosis/Tubular Atrophy. Ultrasound Med Biol. 2015;41:2631-9. doi: 10.1016/j.ultrasmedbio.2015.06.009. PubMed PMID: 26219696.

25. Sommerer C, Scharf M, Seitz C, Millonig G, Seitz $\mathrm{HK}$, Zeier M, et al. Assessment of renal allograft fibrosis by transient elastography. Transpl Int. 2013;26:545-51. doi: 10.1111/tri.12073. PubMed PMID: 23383606.

26. Abbasian Ardakani A, Mohammadi A, Khalili Najafabad B, Abolghasemi J. Assessment of Kidney Function After Allograft Transplantation by Texture Analysis. Iran J Kidney Dis. 2017;11:157-64. PubMed PMID: 28270649.

27. Wybraniec MT, Bożentowicz-Wikarek M, Chudek J, Mizia-Stec K. Pre-procedural renal resistive index accurately predicts contrast-induced acute kidney injury in patients with preserved renal function submitted to coronary angiography. The International Journal of Cardiovascular Imaging. 2017;33:595-604.

28. Komuro K, Yokoyama N, Shibuya M, Soutome K, Hirose M, Yonezawa K, et al. Associations between increased renal resistive index and cardiovascular events. J Med Ultrasonics. 2016;43:263-70. doi: 10.1007/s10396-015-0680-y. PubMed PMID: 27033870.

29. Yazici B, Oral A, Gokalp C, Akgün A, Toz H, Ozbek $\mathrm{SS}$, et al. Evaluation of renal transplant scintigraphy and resistance index performed within 2 days after transplantation in predicting long-term graft function. Clin NuCl Med. 2015;40:548-52. doi: 10.1097/rlu.0000000000000789.

30. Peng L, Zhong T, Fan Q, Liu Y, Wang X, Wang L. Correlation analysis of renal ultrasound elastography and clinical and pathological changes in patients with chronic kidney disease. Clin Nephrol. 2017;87:293-300. doi: 10.5414/CN108866. PubMed PMID: 28332473.

31. Lee J, Oh YT, Joo DJ, Ma BG, Lee AL, Lee JG, et al. Acoustic Radiation Force Impulse Measurement in Renal Transplantation: A Prospective, Longitudinal Study With Protocol Biopsies. Medicine. 2015;94:e1590. doi: 10.1097/ MD.0000000000001590. PubMed PMID: 26426636. PubMed PMCID: PMC4616853.

32. Bota S, Bob F, Sporea I, Sirli R, Popescu A. Factors that influence kidney shear wave speed assessed by acoustic radiation force impulse elastography in patients without kidney pathology. Ultrasound Med Biol. 2015;41:1-6. doi: 10.1016/j.ultrasmedbio.2014.07.023. PubMed PMID: 25438855.

33. Randers E, Erlandsen EJ. Serum cystatin C as an endogenous marker of the renal function--a review. Clin Chem Lab Med. 1999;37:389-95. doi: 10.1515/CCLM.1999.064. PubMed PMID: 10369108.

34. Gökkuşu CA, Özden TA, Gül H, Yıldız A. Relationship between plasma Cystatin $C$ and creatinine in chronic renal diseases and Tx-transplant patients. Clin Biochem. 2004;37:94-7. doi: 10.1016/j.clinbiochem.2003.10.009.

35. Van Acker BA, Koomen GC, Koopman MG, De Waart DR, Arisz L. Creatinine clearance during cimetidine administration for measurement of glomerular filtration rate. Lancet. 1992;340:1326-9. PubMed PMID: 1360044.

36. Andreev E, Koopman M, Arisz L. A rise in plasma creatinine that is not a sign of renal failure: which drugs can be responsible? J Intern Med. 1999;246:247-52. PubMed PMID: 10475992. 